\title{
Discussion: The question of structural form: educational aspects
}

Wanda J. Lewis MSC, PhD, CEng, FICE

Professor, School of Engineering, University of Warwick, Warwick, UK
H. B. Warburton BSC, DIC, CEng, FICE

Retired, Formerly Robin Webb Consulting Ltd, Colchester, UK

\section{Contribution by H. B. Warburton}

The contributor would like to address the discussion first in terms of the educational theme. Then the context of flexible structural form will be addressed.

As regards the educational theme, in her introductory remarks on structural form (Lewis, 2012), Dr Lewis has placed emphasis on the attention required when examining a potential for failure. The care needs to be of a high order, higher indeed than is commonly accepted in ordinary design. It is clear that a young engineer who seeks to practice forensics must first aim to become qualified to a level similar to that of the author. Typically this must mean a fellowship, together with a doctorate and the corresponding breadth of experience.

The arrival of this new journal, as part of ICE Proceedings, has brought into being an excellent medium which will appeal to those at the higher levels of capability.

Turning now to the example of a flexible structural form, namely London Millennium footbridge, the author has made it clear that a serious potential failure had to be forestalled when this bridge was first put into service. This was achieved by the retro-fit of ' $V$ '-shaped (chevron) bracings in the deck, fitted with viscous dampers, and the addition of tuned-mass dampers at particular supports.

The London Millennium footbridge has non-standard features, indicated below, which render it inappropriate to draw upon the traditional guidelines for a normal suspension bridge (as derived from the George Washington Bridge, New York, 1931 and Golden Gate Bridge, which was itself affected by vibrations).

(a) The shallow 'dip' of the main cables in London Millennium footbridge was made possible by the use of high pre-tensions.

(b) The cables are curved horizontally $(13.4 \mathrm{~m}$ spread at abutments, reducing to $10 \cdot 0 \mathrm{~m}$ at mid-point).

(c) The London Millennium footbridge cannot carry point loads. A small vertical load, off-centre, would tighten the cables on that side and slacken those opposite. Horizontal components then give rise to (barely perceptible) lateral movements. However, pedestrians might walk 'in time'. This is synchronous lateral excitation, currently countered by dampers.

(d) The London Millennium footbridge does not have a stiffening girder. The deck is not significant, being made of tongued and grooved aluminium components supported on tubes. The main stiffness is derived from the cables, which were specially fabricated, using interlocking strands.

(e) A measure of the progress in the use of computers up to 1996 is that reliance was placed upon a static stiffness analysis for the structure, including piers, cables, anchorages, deck and approaches, all in three dimensions. The necessary dynamic computations were by specialists, called in after the serious vibrations became manifest.

The final cost of London Millennium footbridge has to be surmised, but is probably around $£ 30$ million, not counting professional fees. It is thought that a conventional but excellent 'engineer's bridge' might have been built for $£ 15$ million, all-in.

The London Millennium footbridge now appears to be performing well enough, without major maintenance, but it is doubtful whether this type of design could be used again, without an exhaustive forensic study and report. Nevertheless it is likely that a future market may exist, to create a network of cycle-ways at high level.

\section{Author's reply}

Mr Warburton's contribution to the discussion of the paper is very welcome; it adds valuable information on the London Millennium footbridge.

The author chose the London Millennium footbridge example to illustrate two points: (a) that the overall geometry of the cable (measured by span/dip ratio) does affect structural efficiency, and (b) that equal length cables supporting the structure behave like guitar strings and, therefore, are susceptible to polarised vibration feedback. 
With regard to point (a), the deck of the bridge is resting on a series of cradle arms, which, in turn, are suspended from the bridge cables at regular intervals. Where the arms connect to the cables, they apply point loads (just as hangers in a suspension bridge do). For simplicity, only one point load was shown in Figure 1(b) to illustrate the resolution of forces and demonstrate that a cable with a low dip cannot transfer vertical loading efficiently. This point applies to any cable-supported structure. The LMF is loaded predominantly in a vertical direction (although, ironically, it was a small horizontal load component that caused stability problems), and for this (vertical) loading, the curvature of the cables in the horizontal plane is not relevant. Figure 1(b) offers a simple explanation as to why extremely high tensions developed in the bridge cables. In fact, tensions exceeded the capability of commonly manufactured products and, as $\mathrm{Mr}$ Warburton correctly states, the cables had to be specially fabricated.

With regard to point (b), the author's understanding is that the London Millennium footbridge was checked for vertical vibrations only, using existing codes. Subsequently, external consultants were called to model the problem of synchronous lateral excitation, and the final solution was provided by dampers.

The example of the London Millennium footbridge highlights the problem of a compromise between structural efficiency/ safety and aesthetics/function that needs to be addressed by any design. In this case, one wonders whether the compromise has gone too far in favour of the latter, that is, provision of an unobstructed view of the river and surrounding landmarks. This case raises a further question of an unequal partnership between an engineer and an architect.

\section{REFERENCE}

Lewis WJ (2012) The question of structural form: educational aspects. Proceedings of the Institution of Civil Engineers Forensic Engineering 165(3): 131-141.

\section{WHAT DO YOU THINK?}

To discuss this paper, please email up to 500 words to the editor at journals@ice.org.uk. Your contribution will be forwarded to the author(s) for a reply and, if considered appropriate by the editorial panel, will be published as discussion in a future issue of the journal.

Proceedings journals rely entirely on contributions sent in by civil engineering professionals, academics and students. Papers should be 2000-5000 words long (briefing papers should be 1000-2000 words long), with adequate illustrations and references. You can submit your paper online via www.icevirtuallibrary.com/content/journals, where you will also find detailed author guidelines. 